\title{
Physically motivated X-ray obscurer models
}

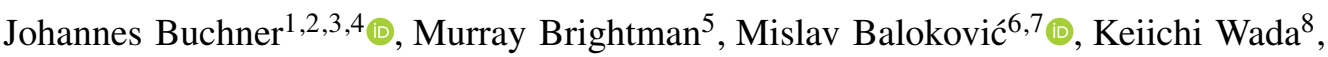 \\ Franz E. Bauer ${ }^{2,4,9}$, and Kirpal Nandra ${ }^{1} \odot$
}

\author{
1 Max Planck Institute for Extraterrestrial Physics, Giessenbachstrasse, 85741 Garching, Germany \\ 2 Pontificia Universidad Católica de Chile, Instituto de Astrofísica, Casilla 306, Santiago 22, Chile \\ e-mail: johannes . buchner . acad@gmx . com \\ 3 Excellence Cluster Universe, Boltzmannstr. 2, 85748 Garching, Germany \\ 4 Millenium Institute of Astrophysics, Vicuña MacKenna 4860, 7820436 Macul, Santiago, Chile \\ 5 Cahill Center for Astrophysics, California Institute of Technology, 1216 East California Boulevard, Pasadena, CA 91125, USA \\ 6 Yale Center for Astronomy \& Astrophysics, 52 Hillhouse Avenue, New Haven, CT 06511, USA \\ 7 Department of Physics, Yale University, PO Box 2018120, New Haven, CT 06520, USA \\ 8 Kagoshima University, Kagoshima 890-0065, Japan \\ 9 Space Science Institute, 4750 Walnut Street, Suite 205, Boulder, CO 80301, USA
}

Received 20 December 2018 / Accepted 1 June 2021

\begin{abstract}
Context. The nuclear obscurer of active galactic nuclei (AGN) is poorly understood in terms of its origin, geometry, and dynamics. Aims. We investigate whether physically motivated geometries emerging from hydro-radiative simulations can be differentiated with $\mathrm{X}$-ray reflection spectroscopy.

Methods. For two new geometries, the radiative fountain model and a warped disk, we release spectral models produced with the ray tracing code XARS. We contrast these models with spectra of three nearby AGN taken by NuSTAR and Swift/BAT.

Results. Along heavily obscured sightlines, the models present different $4-20 \mathrm{keV}$ continuum spectra. These can be differentiated by current observations. Spectral fits of the Circinus Galaxy favour the warped disk model over the radiative fountain, and clumpy or smooth torus models.

Conclusions. The necessary reflector $\left(N_{\mathrm{H}} \geq 10^{25} \mathrm{~cm}^{2}\right)$ suggests a hidden population of heavily Compton-thick AGN amongst local galaxies. X-ray reflection spectroscopy is a promising pathway to understand the nuclear obscurer in AGN.
\end{abstract}

Key words. galaxies: active - accretion, accretion disks - methods: numerical - X-rays: general - radiative transfer - scattering

\section{Introduction}

Most active galactic nuclei (AGN) are obscured by dense gas and dust with column densities of $N_{\mathrm{H}}=10^{22-25} \mathrm{~cm}^{-2}$ (e.g. Ueda et al. 2003; Buchner et al. 2015). Of this, galaxy-scale gas provides at most a minority of this obscuration at moderate column densities $N_{\mathrm{H}}=10^{22-23.5} \mathrm{~cm}^{-2}$ (e.g., Buchner \& Bauer 2017). The predominant obscurer is thought to be a parsecscale circum-nuclear structure (see e.g., Antonucci 1993; Netzer 2015; Ramos Almeida \& Ricci 2017, for reviews). However, the geometry, substructure, and physical origin of this component is still unclear and heavily debated.

Already early modelling efforts (see, e.g., Matt et al. 2000, and references therein) revealed that the unresolved X-ray continuum of AGN is composed of a power law source (with a highenergy cut-off seen in some sources) that is photo-electrically absorbed. The absorbing screen primarily suppresses soft photons, bending the power law downwards towards soft energies. This suppression reveals additional, secondary components interpreted as ionised and cold gas reprocessing and reflecting the primary power law emission. The ionised reprocessing, also called the warm mirror, can be interpreted as Thomson scattering off ionised, stratified volume-filling gas, potentially in the narrow-line-region (e.g., Turner et al. 1997; Bianchi et al. 2006, and references therein). This spectral feature is to first order a copy of the intrinsic power law, with a normalisation approximately $0.1-10 \%$ of the intrinsic emission, and common in obscured AGN (e.g., Rivers et al. 2013; Buchner et al. 2014; Brightman et al. 2014; Ricci et al. 2017).

The interaction in cold reprocessing is more complex. About a third of all AGN have line-of-sight (LOS) column densities $N_{\mathrm{H}}$ in excess of the Compton-thick limit $\left(N_{\mathrm{H}}=\sigma_{\mathrm{T}}^{-1} \approx\right.$ $1.5 \times 10^{24} \mathrm{~cm}^{-2}$; e.g., Buchner et al. 2015; Ricci et al. 2015). This implies that Compton scattering is an important process. $\mathrm{X}$-rays from the corona recoil off distant, cold, dense gas, and in the process lose energy and change direction. The Compton scattered spectrum depends on the geometry and density of the reflecting cold matter. The reflection was first modelled as simple, semi-infinite slabs (e.g., Magdziarz \& Zdziarski 1995; Matt et al. 1999). Slabs are designed to mimic the accretion disk and produces a very hard spectrum (negative effective photon index), decorated with fluorescent lines such as $\mathrm{Fe} \mathrm{K} \alpha$ and absorption edges. An important feature is excess emission around $10-50 \mathrm{keV}$, the 'Compton hump'. As knowledge and capabilities in spectral and spatial resolution progressed, more realistic modelling was needed, allowing refined parameter constraints and interpretations (e.g., models by Murphy \& Yaqoob 2009; Brightman \& Nandra 2011; Ikeda et al. 2009). Today, the obscurer geometry is still uncertain. This is partly because the observed X-ray emission can be contaminated by nearby X-ray binaries, supernova remnants and ultra-luminous X-ray sources (e.g., Arévalo et al. 2014), and because extended emission can 
contribute significantly to the $\mathrm{Fe} \mathrm{K} \alpha$ flux (e.g., Bauer et al. 2015). Even with these taken into account, detailed study of the nearby Compton-thick AGN NGC 1068 reveals multiple cold reflectors of different column densities (Bauer et al. 2015). Also, there appears to be significant diversity between sources (e.g., Baloković et al. 2018). Other studies find that the reflection in heavily obscured sources is best-fitted by a face-on orientation of a torus-shaped geometry (e.g., Baloković et al. 2014; Gandhi et al. 2014). Finally, observations of variability in measured column density (e.g., Risaliti et al. 2002; Markowitz et al. 2014) indicates that the obscurer is clumpy and subsequently, the first clumpy models have been developed (e.g., Nenkova et al. 2008; Furui et al. 2016; Liu \& Li 2014; Buchner et al. 2019; Tanimoto et al. 2019). The applied X-ray obscurer models are perhaps the simplest geometries for a unified obscurer. However, it remains unclear which specific physical mechanisms maintain the high covering factors of these geometries and where the obscuring gas originates.

The above considerations indicate that our understanding of the obscurer geometry is still incomplete, in part due to a dearth of self-consistent absorber and reflector models with physical motivations. In the last decade, several theoretical models based on radiative hydro-dynamic simulations have been developed, which attempt to explain the origin and stability of the obscurer. The high covering is achieved either by bringing in host galaxy scale gas from random orientations (e.g., Hopkins et al. 2012; Gaspari et al. 2015), by black hole and star formation feedback processes in the inner hundred parsec (e.g., Wada 2012; Schartmann et al. 2018), or by the black hole accretion system in isolation (e.g., Chan \& Krolik 2016; Dorodnitsyn \& Kallman 2017; Williamson et al. 2019; Mościbrodzka \& Proga 2013). However, such models have not yet been tested against X-ray reflection spectroscopy. We investigate the spectral signatures of different physically motivated geometries, and whether they can be differentiated by current X-ray instruments. In Sect. 2.1, we develop an open-source Monte Carlo code that can be used to simulate the irradiation of arbitrary grid geometries and produce X-ray spectra. We investigate two new X-ray spectral predictions based on physical models, presented in Sect. 3. The geometries were dynamically created in the environment of super-massive black holes by effects like gravity and radiation pressure. Section 4.1 presents the emerging X-ray spectra, and Sect. 4.3 validates them against $\mathrm{X}$-ray observations. Section 5 discusses the physical implications of the results and suggests future observational tests.

\section{Methodology}

\subsection{Simulations}

Monte Carlo simulations are used to compute the emerging X-ray spectrum for several geometries of interest. For this, we developed a modular, Python-based simulation code XARS (X-ray Absorption Re-emission Scattering, Buchner et al. 2019), which is publicly available ${ }^{1}$. The simulation method is described in detail in Brightman \& Nandra (2011). Briefly, a point-source emits photons isotropically from the centre of the obscurer geometry. Photo-electric absorption, Compton scattering and line fluorescence are simulated self-consistently as photons pass through matter, altering the direction and energy of the photons or absorbing them. For simplicity and to compare with other works, solar abundances from Anders \& Grevesse (1989) are assumed

\footnotetext{
https://github.com/JohannesBuchner/xars
}

with cross-sections from Verner et al. (1996). XARS computes the Green's functions at each defined energy grid point and viewing angle, onto which an input photon spectrum, e.g., a powerlaw, can be applied, and transformed into an Xspec grid model.

Users of XARS specify the geometry of the obscurer by implementing how far, starting from a given location and direction, photons can propagate through the medium. For this work we additionally implemented photon propagation through 3-dimensional density grids, such as those produced from hydrodynamic simulations. Our parallelised and optimised C implementation is based on the 3D Digital Differential Analyser (Fujimoto et al. 1986) and publicly available within the LightRayRider ${ }^{2}$ library (Buchner et al. 2017) and can easily be used with XARS (examples are included in the XARS documentation). Digital Differential Analysers are a simple but efficient technique to traverse grids, and compute the length before a new grid line is encountered. Assuming constant-density grid cells, this allows us to integrate the column density along the photon path efficiently. The exact technique is described in Appendix A. This enables the computation of X-ray spectra emerging from arbitrary density distributions.

\subsection{Observations}

After presenting the new spectral models in the following sections, preliminary comparisons to observations are made. A rigorous comparison of careful spectral fits to an unbiased sample is left for future work. Here, we test the plausibility of the models on three sources: Circinus Galaxy, NGC 424 and ESO 103-G03. These are bright and well-studied examples of nearby AGN that demonstrate a variety of spectral features of interest in our study. The NUSTAR spectra of the three sources was previously analysed in Buchner et al. (2019). All three show prominent reflection on top of a obscured continuum, but their accretion rate and line-of-sight obscuration is diverse. They were targeted by NUSTAR in single-epoch observations (ObsIds: 60061007002, 60002039002, 60061288002) for 10-60 ks. Spectra were extracted using the NuSTAR data processing tools in the same manner as in Brightman et al. (2015). The NUSTAR spectra are binned to 20 counts per bin and $\chi^{2}$ statistics adopted, which is justified in these high count spectra.

Of our three sources, the closest and best understood is the Circinus Galaxy. One of the model geometries we consider was specifically developed for this galaxy. For this reason, we include a detailed spectral analysis of this source. Within the point spread function of current hard X-ray instruments, extended emission is present (e.g., Bauer et al. 2015; Fabbiano et al. 2018), and below $10 \mathrm{keV}$ components from star formation and X-ray binaries are important (Marinucci et al. 2013; Arévalo et al. 2014). For this reason, we include these components in a joint fit. To focus on the high-energy shape, we consider the NUSTAR spectrum from $8-75 \mathrm{keV}$ and add Swift/BAT spectra from the 105 month data release (Oh et al. 2018). For the other two sources, we only make visual comparisons with some models above $8 \mathrm{keV}$ as a first-order validation, while more detailed analyses are left for a future publication.

\section{Geometries}

Realistic obscurer models need to explain the high obscured fraction in the AGN population. This requires sustaining large covering factor around the X-ray source (see, e.g.,

\footnotetext{
2 https://github.com/JohannesBuchner/LightRayRider
} 


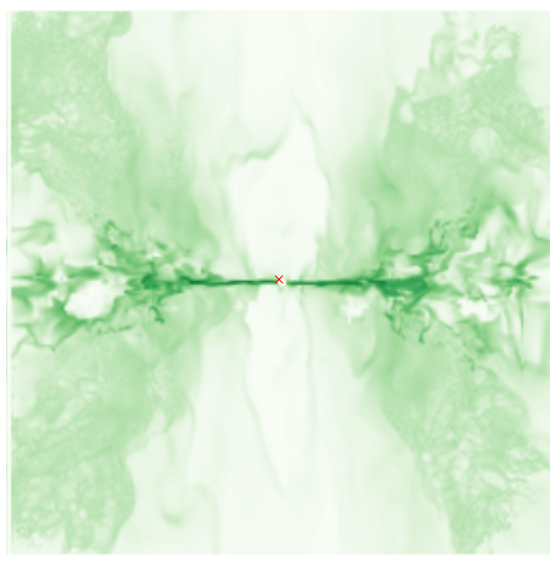

Fig. 1. Geometry of the radiation-driven fountain model of Wada et al. (2016). A cross-section of the density grid is shown with the X-ray emitting corona indicated by the red cross. The side length of the simulation region is $32 \mathrm{pc}$.

Lawrence \& Elvis 2010; Wada 2015; Schartmann et al. 2018). Below we present two physically motivated X-ray obscurer models: A warped disk and the radiative fountain model. In the following section, we discuss the resulting spectra.

\subsection{Wada's radiative fountain model}

Wada (2012) developed a model in which the obscurer is a radiation pressure-driven polar outflow from a black hole accreting within a thick, parsec-scale disk. The outflow falls back akin to a fountain (see Fig. 1) and forms a torus-like shape producing suitably high covering factors (Wada 2015). In Wada et al. (2016), supernova feedback is also included. The evolution of this system was computed with three-dimensional radiation hydrodynamic simulations on a $256^{3}$ cell grid spanning a domain of $(32 \mathrm{pc})^{3}$. As a representation of this model, we use the Wada et al. (2016) geometry from their final simulation time step. Figure 1 shows the geometry for a black hole mass of $M_{\mathrm{BH}}=2 \times 10^{6} M_{\odot}$ and $20 \%$ Eddington rate, suitable for comparison to Circinus (see Wada et al. 2016, 2018a,b; Izumi et al. 2018, for comparison with infrared SEDs, atomic/molecular gas, and ionised gas observations, respectively). The model has a Compton-thick LOS column density only under edge-on viewing angles (consistent with the infrared analysis of Circinus by Wada et al. 2016).

The X-ray spectrum under this geometry is computed by placing the X-ray corona at the centre of the simulations density grid (see Fig. 1). We use XARS to irradiate the geometry with $5 \times 10^{9}$ photons, and capture the energy and direction of X-ray photons escaping to infinity. The direction is used to divide photons by viewing angle in two steps. First, the LOS column density $\left(N_{\mathrm{H}}\right)$ from the corona to infinity in that direction is computed, which defines the first binning axis. Secondly, we further sub-divide by azimuthal angle (face-on to edge-on). This scheme (also in Buchner et al. 2019) has the benefit of separating out the most important observable ( $\operatorname{LOS} N_{\mathrm{H}}$ ), and allowing varying the LOS $N_{\mathrm{H}}$ and viewing angle independently.

\subsection{Warped disk}

Warped, tilted and twisted disks have been proposed as the source of (Compton-thick) obscuration (e.g., Lawrence \& Elvis 2010). Non-planar accretion can cause precessing concentric

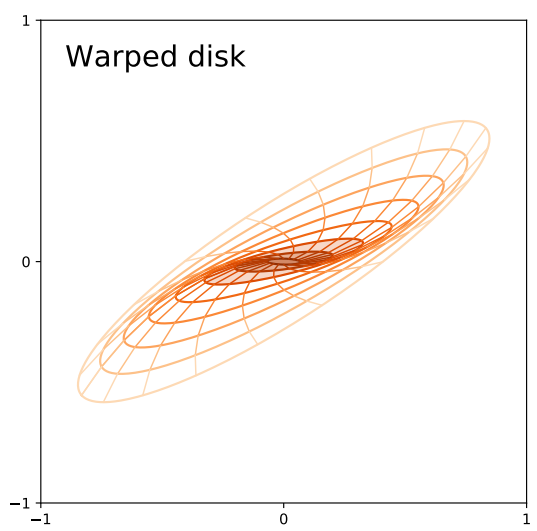

Fig. 2. Warped disk geometry, viewed edge-on. Near the centre the disk is only mildly warped (shaded inner-most two annuli, $r_{\text {warp }} \sim 0.25$ ), and viewing angles are defined relative to there. The outer disk annuli (up to radius $r_{\text {warp }}=1$ ) are warped more strongly and form a wall, suitable for Compton reflection. For our simulation, we set up a grid with $256^{3}$ cells and assign cells intersecting with this shape a Compton-thick column density.

rings of accretion disks (Petterson 1977a). Additionally, the disk emits normal to the local surface, but the received radiation pressure from the centre is misaligned and causes torque, and thus twisted disks (Petterson 1977b). The shape distortion is limited by viscosity, as gas moves radially inward.

Water maser disks are one type of such warped disks known to exist in several AGN. Strongly emitting disk clumps trace out edge-on warped disk structures, e.g., in Circinus (Greenhill et al. 2003). Recently, Jud et al. (2017) developed an infrared model for the warped disk. They demonstrate good fits to infrared photometry and can naturally explain the offset between point source and disk component seen in the highest resolution infrared VLTI observations (Tristram et al. 2014). Water maser disk systems are always found to be heavily obscured (Greenhill et al. 2008; Masini et al. 2016), indicating that the Compton-thick obscurer either is the warped maser disk itself or at least shares the same plane of orientation.

We now simulate such a warped disk obscurer. Pringle (1996) describes the geometry of a warped disk as

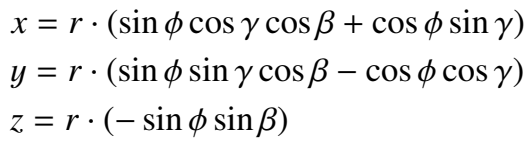

with $\gamma(r)=\sqrt{r}$ and $\beta(r)=\sin (\gamma(r)) / \gamma(r)$, following Maloney et al. (1996). The parametrisation essentially describes a disk ring at radius $r$ across angles $\phi \in[0,2 \pi]$. We set up a computation grid of $256^{3}$ cells and assign a column density of $N_{\mathrm{H}}=10^{25} \mathrm{~cm}^{-2}$ to all grid cells intersecting the warped disk, as well as the cells just below to produce a thickness of at least two grid cells everywhere.

We set up various warp strengths. We let the radii $r$ range from zero (the centre) up to $r_{\text {warp }}=1,1 / 2,1 / 4$ or $1 / 16$. The case of a strong warp $\left(r_{\text {warp }}=1\right)$ is illustrated in Fig. 2 and features a prominent wall. For $1 / 4$ and $1 / 16$, only the flat thin (unwarped) inner disk remains, illustrated as the inner annuli of Fig. 2.

\section{Results}

\subsection{Continuum shapes}

Examples of obscured spectra are presented in Fig. 3. The input photon spectrum is a cut-off powerlaw $E^{-\Gamma} \times \exp \left(-E / E_{\text {cut }}\right)$ with 

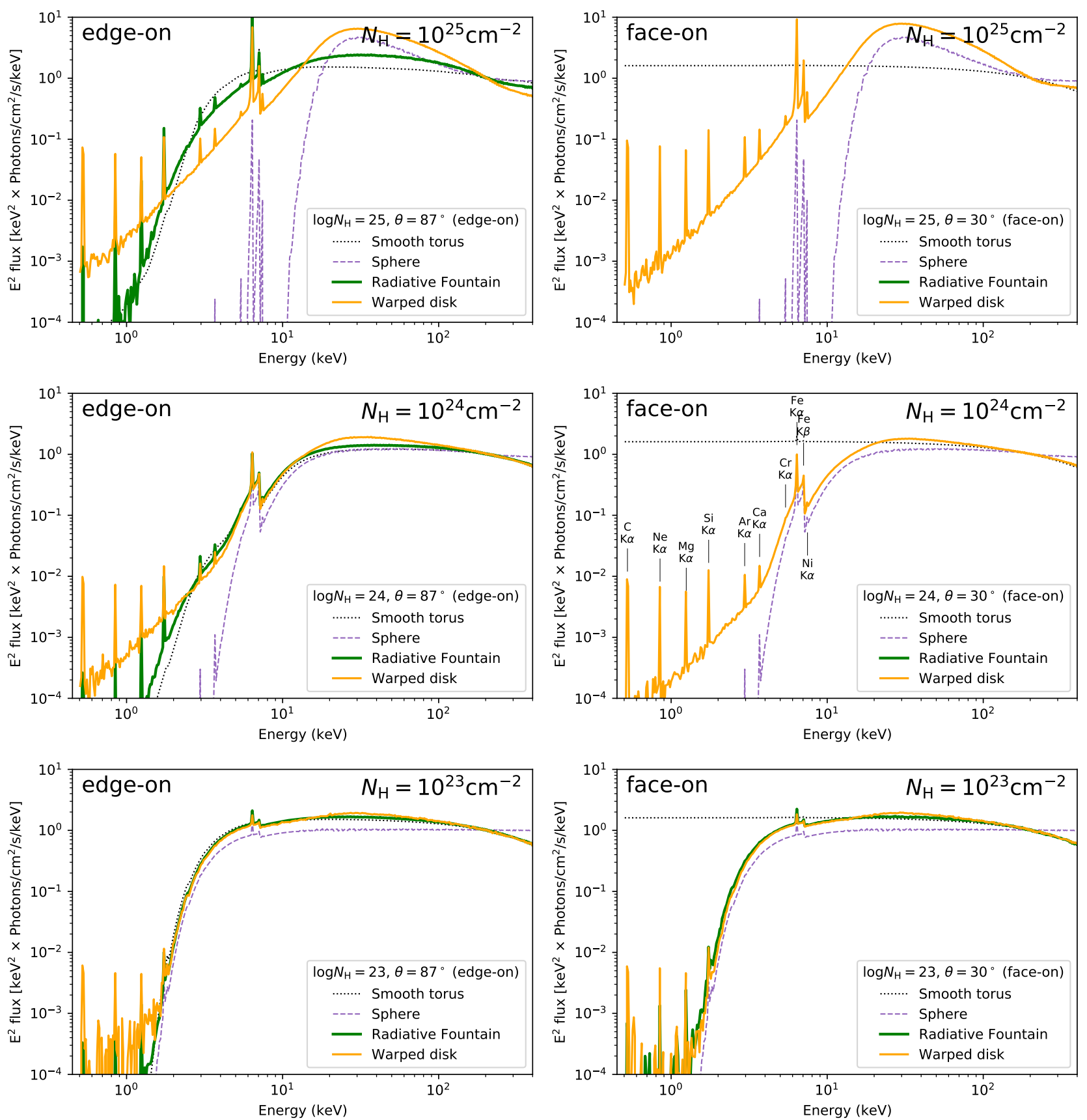

Fig. 3. Model spectra of the radiative fountain model (green) and warped disk model (orange). The rows show different line-of-sight column densities. Left (right) column: a nearly edge-on (face-on) view of the obscurer. For comparison, a spherical obscurer (Brightman \& Nandra 2011) and smooth torus (Murphy \& Yaqoob 2009) are included as purple dashed and black dotted curves, respectively. In low column densities (bottom row) the predictions are similar. Right column: the radiative fountain predictions are absent because near-Compton-thick column densities only occur in the equatorial plane. Similarly, the torus is unobscured from this viewing angle and shows the input powerlaw. The models differ most in the 2-20 keV range under high obscuring column densities (top left panel). The warped disk produces the strongest emission near $20 \mathrm{keV}$ among the models. Models are normalised at $200 \mathrm{keV}$. Centre right panel: included fluorescent lines.

photon index $\Gamma=2$ and exponential cut-off $E_{\text {cut }}=400 \mathrm{keV}$. The panels of Fig. 3 plot the emerging $E^{2} \times$ photon spectrum, split by inclination and LOS column density. For orientation, we include two models based on simple geometries in Fig. 3. The sphere model of Brightman \& Nandra (2011) embeds the $\mathrm{X}$-ray point source in a constant-density sphere. This model completely suppresses photons below $10 \mathrm{keV}$ in Compton-thick sightlines, except for Fe K fluorescence. The smooth torus model of Murphy \& Yaqoob (2009) surrounds the X-ray point source with a constant-density torus, with an opening angle of $60^{\circ}$. Its soft emission is dominated by Compton reflection off the torus. In moderately obscured sightlines (bottom panels of Fig. 3), all models make similar predictions. The sphere model differs slightly from the others, as it does not include an exponential cut-off.

The models differ in the strength of the Compton hump near $10-50 \mathrm{keV}$. The warped disk model shows the strongest emission, while the radiative fountain model has a weak Compton 


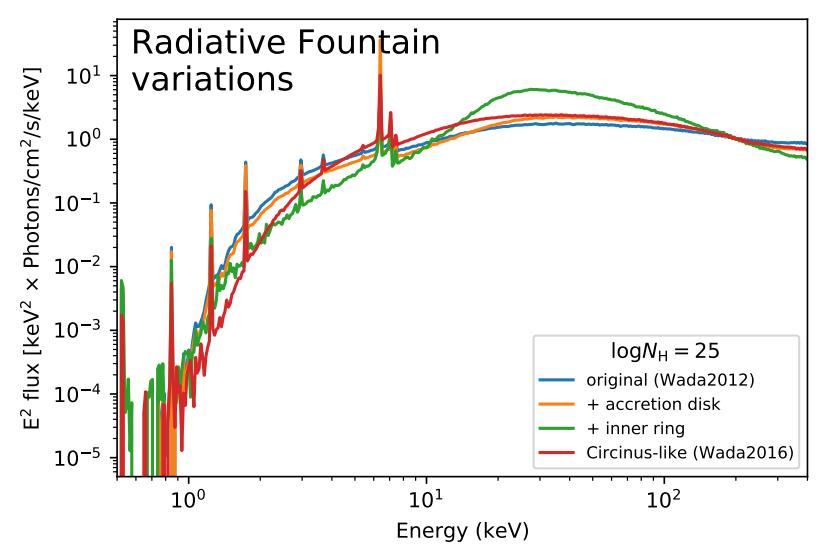

Fig. 4. Variations of the radiative fountain model spectra. The Wada (2012) and Wada et al. (2016) models (blue and red, respectively) differ in black hole mass and supernova feedback. The orange curve includes reflection from an accretion disk. The green curve includes a Comptonthick ring around the central X-ray source, which leads to stronger emission in the $10-50 \mathrm{keV}$ energy range than the original model (blue curve). Models are normalised at $200 \mathrm{keV}$.

hump. This is likely related to the high covering factor of highdensity material in the warped disk that is seen without further obscuration by the observer. The two models bracket the sphere model in terms of the Compton hump strength. Additionally, the hump shapes are different in their turn-over.

The models differ most strongly in the $2-20 \mathrm{keV}$ energy range under Compton-thick LOS (e.g., top left panel). Here the transmitted powerlaw continuum is suppressed, revealing the Compton reflected components and fluorescent lines. In both of our two models, soft photons escape under all viewing angles through Compton scattering.

The spectral slopes in the $2-20 \mathrm{keV}$ energy range are diverse. The spherical obscurer shows the steepest slope among the models. The smooth torus and radiative fountain models show a smooth exponential turn-over, at lower energies than the sphere geometry. In contrast, the warped disk model presents a powerlaw-like continuum in the $2-20 \mathrm{keV}$ energy range with photon index $\Gamma \approx 0.5$. The warped disk produces the strongest emission near $20 \mathrm{keV}$ among the models. Its Compton hump is widest in the radiative fountain model.

We explore these differences further in Figs. 4 and 5. Figure 4 shows the spectra from the models of Wada (2012) and Wada et al. (2016), which differ primarily in that the latter uses a ten times smaller black hole mass and the presence of supernova feedback, both chosen to match the Circinus Galaxy. The X-ray spectral predictions are however of similar shape, characterised by a smoothly bending reflection spectrum in the $2-20 \mathrm{keV}$ energy range. The resulting spectra are very similar across viewing angles, if the LOS column density are kept constant. However, Compton-thick sightlines, as shown in Fig. 4, only exist in equatorial views.

\subsection{Model variations}

Reflection close to the X-ray corona can alter the spectral shape. The accretion disk is a known reflector, and can be modelled by a semi-infinite slab. We replace the input powerlaw source with a powerlaw source and its disk-reflected, angle-averaged spectrum. The disk model spectrum was computed with XARS and presented in the appendix of Buchner et al. (2019), and is consistent with the pexmon disk model (Nandra et al. 2007). The

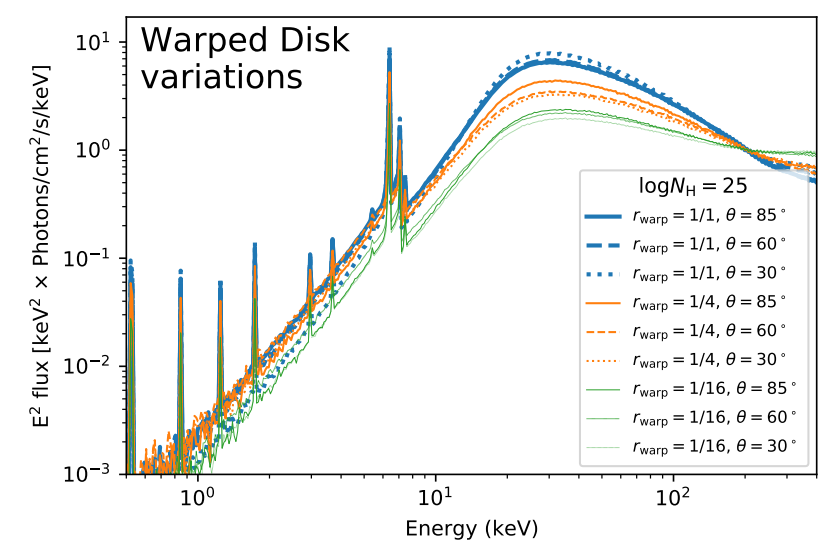

Fig. 5. Variations of the warped disk model. Variations of the inclination angle relative to the innermost annuli are represented by solid, dashed and dotted lines. Variations of the extent of the warp (see Fig. 2) are represented by line strengths. The thickest lines (strong warps) exhibit the strongest emission in the $20-30 \mathrm{keV}$ range. Models are normalised at $200 \mathrm{keV}$.

effect of the accretion disk model can be seen by comparing the orange curve in Fig. 4 with the blue curve. The model with accretion disk (orange) shows a higher Compton hump than the model without (blue). However, the difference is less than a factor of 2. This may be because the disk adds reflection, but does not hinder the powerlaw emission from the X-ray source over half of the sky.

Higher covering factor obscuring structure at small scales have more drastic changes. We experiment how the fountain model would need to be minimally changed to behave like the warped disk model. At the same time, the ultraviolet radiation pressure of the model should remain unaffected. A completely ionised structure between accretion disk and $<0.1$ pc scales may be a viable solution. This could be similar to the dense, inner shielding gas of wind simulations by Gallagher et al. (2015), and could also be interpreted as a sub-parsec scale warped disk, or the broad line region. For simplicity, we achieve a toroid with a opening angle of approximately $60^{\circ}$, by setting the eight spaxels closest to the X-ray emitter in the equatorial plane to Comptonthick column densities. The emerging spectrum is shown in green in Fig. 4. The spectral shape is dramatically different, showing a much stronger Compton hump. It is very similar to the warped disk model (see top left panel of Fig. 3).

Variations of the warped disk models are shown in Fig. 5. The most important parameter is the extent of the warp (increasing with line thickness). For stronger warps (see visualisation in Fig. 2), the increased area available for Compton reflection strengthens the Compton hump emission near $20 \mathrm{keV}$. The viewing angle (defined relative to the innermost annuli) has a minor effect, because of the twisting shape of this geometry.

\subsection{Plausability of models}

The model diversity shown in the previous section indicates that in the Compton-thick regime, hard X-ray spectra can differentiate model geometries. As outlined in Sect. 2.2, we explore the plausibility of our models by projecting them through the $N U S T A R$ response and comparing against a few, high-quality observations. Typical AGN source parameters (a powerlaw continuum with photon index $\Gamma=2$ ) are assumed, and the lineof-sight obscuration is varied. As contaminating components all decline steeply with energy, therefore we normalise each model 

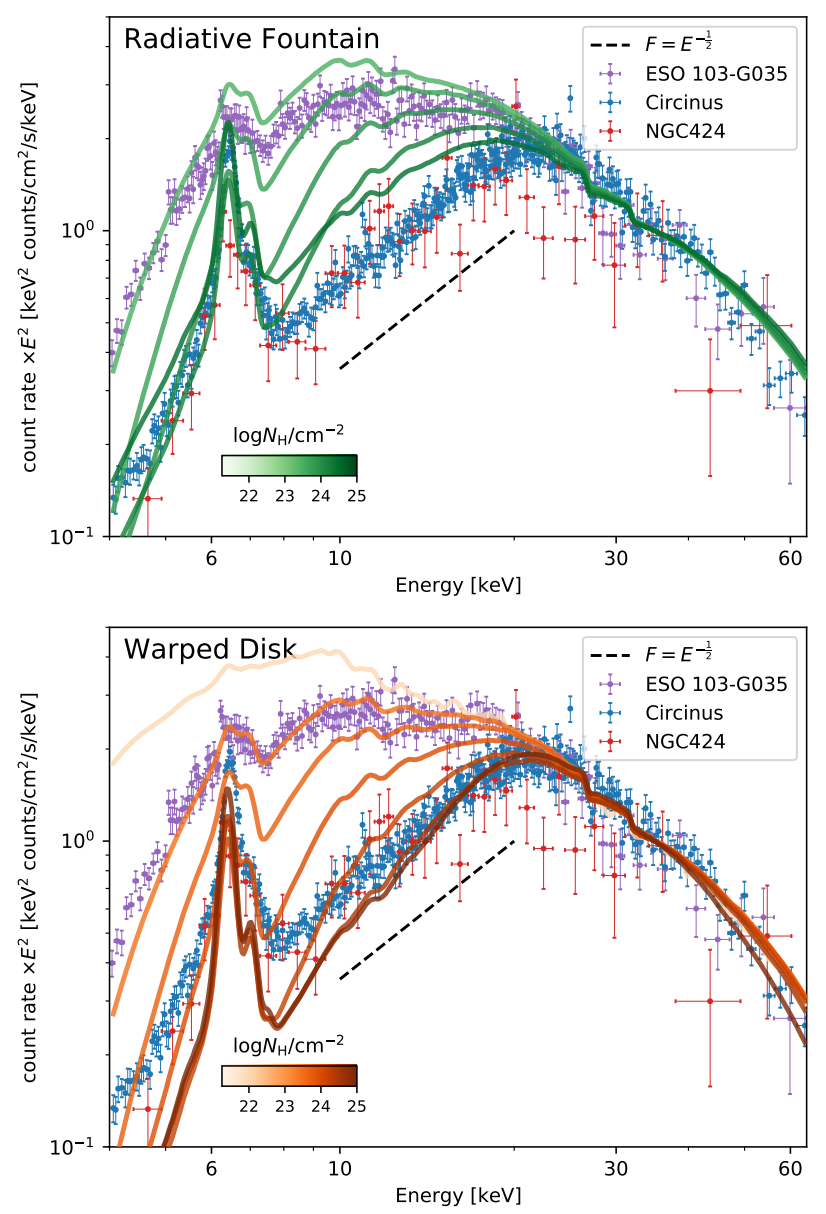

Fig. 6. Comparison of radiative fountain model (top panel) and warped disk model (bottom) to three observed spectra of AGN (error bars). Each panel shows one fixed model geometry. Model instances with photon index $\Gamma=2$ and LOS column densities of $\log N_{\mathrm{H}}=$ $20,23.5,23.75,24,24.25,24.5,26$ (top to bottom curves) are folded through the three instrument responses (near-overlapping groups of curves). Data and models are normalised at $35 \mathrm{keV}$. Top panel: the radiative fountain models show an excess near $10 \mathrm{keV}$ compared to the data. Bottom panel: the warped disk model follows the ESO 103-G035 data closely, and also reproduces the Circinus data. The models underproduce the data at $4-8 \mathrm{keV}$.

with the data at the high energy end and accept models that do not overpredict the data.

Figure 6 shows three NuSTAR spectra of nearby AGN. The high-quality data reveal the reflector in great detail. The top panel of Fig. 6 compares spectra from the radiative fountain model (green) to the data. An input power law with photon index of $\Gamma=2$ is seen through varying layers of column density (green curves). The data of ESO 103-G035 mostly match some of the top model curves, but the $8-10 \mathrm{keV}$ data are underpredicted. This could be accommodated by additional soft energy emission components. The situation is more discrepant when considering the Circinus Galaxy. While the data show a powerlaw-like increase from 8 to $20 \mathrm{keV}$, the models present a wide hump from 8 to $20 \mathrm{keV}$. The models overpredict the data at $10-25 \mathrm{keV}$ even at the highest column densities (lowest curve). A better result may be possible, if an additional reflector was included (see Fig. 4). The data of NGC 424 mimic those of the Circinus Galaxy, but have larger uncertainties.

The bottom panel of Fig. 6 compares spectra from the warped disk model (orange) to the data. Here, the data of

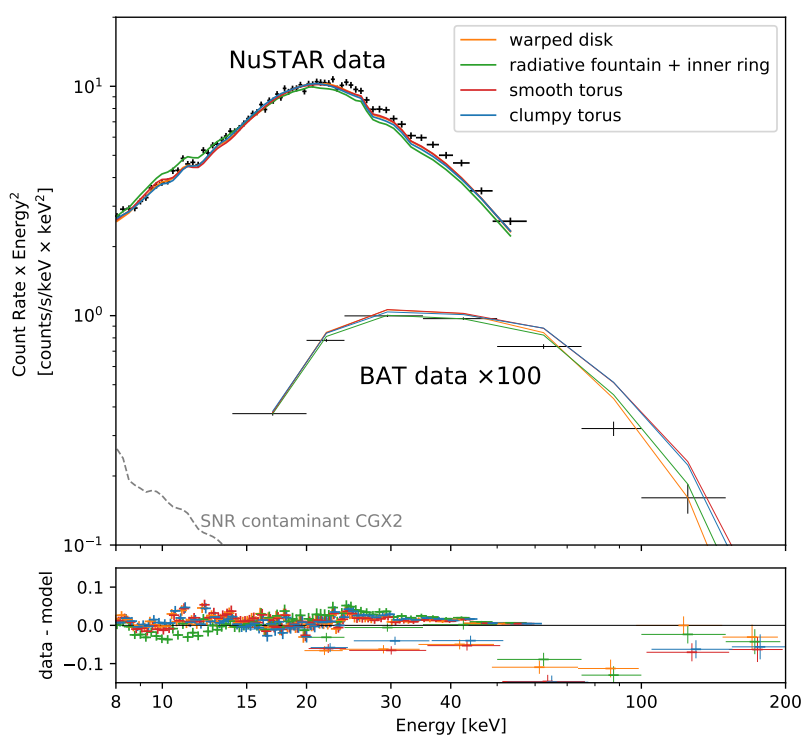

Fig. 7. Circinus hard X-ray spectrum with fitted models. The $\mathrm{NuS}$ TAR $8-75 \mathrm{keV}$ (here heavily rebinned just for visualisation) and BAT $15-150 \mathrm{keV}$ spectra were fitted with four models. The warped disk model yields the lowest $\chi^{2}$.

ESO 103-G035 are directly reproduced by the model, in terms of the $3-6 \mathrm{keV}$ slope, the $8-20 \mathrm{keV}$ shape and the Compton hump strength. The same is true for NGC 424. For the Circinus Galaxy, the data can be matched well from $10 \mathrm{keV}$ upwards. At energies below $10 \mathrm{keV}$, an additional contribution appears to be needed. This is expected. In Buchner et al. (2019) and many other works, an additive powerlaw component has been employed for this purpose. The photon index $\Gamma$ has not been tuned, which could lead to even better agreement. The projection of the warped disk model onto the data is encouraging. To test this more thoroughly, the multiple spectral components and their model parameters have to be fitted jointly.

\subsection{Test case: Spectral fit to Circinus}

Finally, a full spectral fit is performed to the data from the Circinus Galaxy. This object is most suitable, because the hydrodynamic simulations of the radiative fountain model have been tailored to its parameters (accretion rate and black hole mass). Appendix B makes comparisons to the other two galaxies considered in this work. The observed spectrum from NuSTAR and Swift/BAT, presented in Fig. 7, also exhibits high photon count statistics. For clear presentation, the NUSTAR spectra were summed and rebinned to 1000 counts per bin, but for fitting we use a spectrum binned to 20 counts per bin.

Four spectral models of the obscurer geometry are considered. From this work, the warped disk model and the radiative fountain model with the inner ring modification are included. The smooth torus geometry of Murphy \& Yaqoob (2009) and the clumpy torus of Liu \& Li (2014) are also considered. In addition to a powerlaw source processed by an obscurer model, two further components are included. A secondary, unobscured powerlaw is included with the same photon index and a normalisation $f_{\text {scat }}$ of up to $10 \%$ of the primary powerlaw normalisation. The CGX2 supernova remnant contamination has been included as a frozen mekal component with values taken from Arévalo et al. (2014). The spectrum was fitted with all parameters controlling the spectral shape $\left(N_{\mathrm{H}}, \Gamma, f_{\text {scat }}\right)$ free to vary. The normalisation in 
Table 1. Best-fit models parameters for the Circinus Galaxy.

\begin{tabular}{lccccr}
\hline \hline Model geometry & $\begin{array}{c}N_{\mathrm{H}} \\
\left(10^{24} \mathrm{~cm}^{-2}\right)\end{array}$ & $\Gamma$ & $\begin{array}{c}f_{\text {scat }} \\
(\%)\end{array}$ & $\begin{array}{c}\theta_{\text {incl }} \\
\left({ }^{\circ}\right)\end{array}$ & $\chi^{2} /$ d.o.f. \\
\hline (Various, in Arévalo et al. 2014) & $3-20$ & $1.6-2.5$ & & $\sim 75$ & - \\
Smooth torus & $6.4 \pm 0.4$ & $\geq 2.6$ & $\leq 5 \times 10^{-3}$ & $81.8 \pm 0.5$ & $1502 / 1463$ \\
Clumpy torus & $\geq 9.93$ & $2.43 \pm 0.03$ & $30 \pm 6 \times 10^{-2}$ & $\geq 85$ & $1523 / 1462$ \\
Warped disk & $12.61 \pm 3.30$ & $2.00 \pm 0.01$ & $2.9 \pm 1.3 \times 10^{-3}$ & $51 \pm 21$ & $1432 / 1462$ \\
Radiative fountain + inner ring & $16 \pm 1$ & $1.97 \pm 0.007$ & $\leq 3 \times 10^{-4}$ & $82 \pm 8$ & $1451 / 1463$ \\
\hline
\end{tabular}

each data set (FPMA, FPMB, BAT) were left free to vary independently, to account for cross-calibration factors.

We report the most important spectral parameters in Table 1. The fit $\chi^{2}$ statistic is best in the warped disk model $\left(\chi^{2} /\right.$ d.o.f. $=$ $1432 / 1462)$. The radiative fountain model is a good fit when an inner ring is inserted $\left(\chi^{2} /\right.$ d.o.f. $\left.=1451 / 1463\right)$, but not without $\left(\chi^{2} /\right.$ d.o.f. $\left.=4392 / 1463\right)$. In both models the photon index is $\Gamma=2$, close to typical values in the population $(\Gamma=1.95 \pm 0.15$, Nandra \& Pounds 1994; Ricci et al. 2017). The clumpy torus $\left(\chi^{2} /\right.$ d.o.f. $\left.=1523 / 1462\right)$ and the smooth torus $\left(\chi^{2} /\right.$ d.o.f. $=$ $1502 / 1463)$ do not fit the data as well. The NUSTAR data force these models to high, atypical photon indices $(\Gamma>2.4)$, which are disfavoured by the Swift/BAT data $(\Gamma=2.09 \pm 0.02$ in $\mathrm{Oh}$ et al. 2018). All models indicate very high column densities $\left(N_{\mathrm{H}} \geq 10^{25} \mathrm{~cm}^{-2}\right)$. For reference, we also list the parameters found in the recent detailed analysis of Arévalo et al. (2014), which considered a multitude of models, including the smooth torus model.

\section{Discussion and conclusion}

In this work we have extended the open source XARS Monte Carlo spectra simulation software to irradiate arbitrary density grids. We explore physically motivated geometries, including Wada's radiative fountain model and warped disks. This allows for the first time investigation of gas geometries predicted by hydro-dynamic simulations. We publish the spectra as Xspec table models ${ }^{3}$.

\subsection{Geometry information in X-ray spectra}

Different geometries of AGN obscurers predict very different $\mathrm{X}$-ray spectra. In particular, powerful indicators of the obscurer geometry in heavily obscured AGN are illustrated by the top left panel of Fig. 3. These include the continuum slope just below the Fe $\mathrm{K}$ emission feature $(2-6 \mathrm{keV})$, the bend of the continuum between the Fe K edge and the Compton hump (8-20 keV). Below we discuss how different geometries produce different shapes.

Spherical, completely covering obscurers predict virtually no escape of continuum photons below $10 \mathrm{keV}$. Such geometries have been suggested for merging galaxies, but are difficult to test because of the low-energy contamination by stellar processes in these often star-bursting galaxies (e.g., Arp 220, see Teng et al. 2015).

The reflection processes are more elaborate in other geometries. Warped disks, sightlines through the disk heavily suppress the continuum, while an elevated far side can provide an unabsorbed reflection spectrum. This behaviour resembles smooth

\footnotetext{
https://github.com/JohannesBuchner/xars
}

torus geometries (Murphy \& Yaqoob 2009), where the far side also provides substantial reflection.

The warped disk structure requires much less mass than a smooth torus, and is dynamically stable. The extent of the warp is related to the covering factor and influences the strength of the Compton hump emission (see Fig. 5).

The radiative fountain model is more complex. A mixture of constant-density spheres can help understand the emerging spectral shape. The reflection off a single, spherical blob in the 8-20 keV band is shown in Fig. 8 (middle four cases, from the appendix of Buchner et al. 2019). High-density blobs show a steeply rising spectrum, while low-density blobs also let lowenergy photons escape. Combining low, intermediate and highdensity blobs is shown in the bottom of Fig. 8. The mixture of these reflection spectra leads to a wide, smooth hump. Such a spectrum emerges from the radiative fountain model (dotted green curve). In that model, photons interact with a wide range of mostly Compton-thin densities as they travel towards infinity. The truncation towards soft energies in Fig. 3 can be explained by photo-electric absorption by a Compton-thin gas envelope.

\subsection{The obscuring structure of nearby galaxies}

High-quality high-energy spectra from nearby galaxies enable differentiating obscurer geometries. Indeed, the warped disk model was selected as the best fit to the Circinus Galaxy. However, a more generic conclusion can be drawn. The observed spectrum evinces a power law of $E^{-\frac{1}{2}}$ in the $8-20 \mathrm{keV}$ energy window (dashed line in Fig. 6). A preliminary analysis of more AGN observed with NuSTAR indicates that such spectral shapes occur only in rapidly accreting AGN with Eddington accretion ratios of $\dot{M} / \dot{M}_{\text {Edd }} \gtrsim 20 \%$. The examples in Fig. 8 illustrate that such photon flux shapes are only possible with a bimodal distribution of blob densities, including heavily Compton-thick blobs and thinner blobs $N_{\mathrm{H}} \lesssim 10^{23} \mathrm{~cm}^{-2}$. For the radiative fountain model, this suggests that if the thick disk was denser, the model may follow the data better. Another way to achieve this result, illustrated by the orange curve in Fig. 4, is to insert an inner highcovering obscuring structure. This effectively prevents X-ray reflection from the thick disk and alters the spectrum substantially. A very similar conclusion was reached by Buchner et al. (2019), who inserted a inner ring into a clumpy obscurer model.

Figure 9 illustrates the generic recipe to produce the observed shapes. Firstly, the observed spectrum is reflectiondominated, therefore the sightline between observer and the X-ray corona features the Compton-thick obscuration. Secondly, Compton-thick reflecting surfaces with high covering factors are required to have unobscured sightlines both to the observer and the X-ray corona. The accretion disk is unsuitable for this (see Fig. 4). This is because in non-relativistic, flat accretion disks the disk reflection and powerlaw both pass through the absorber 


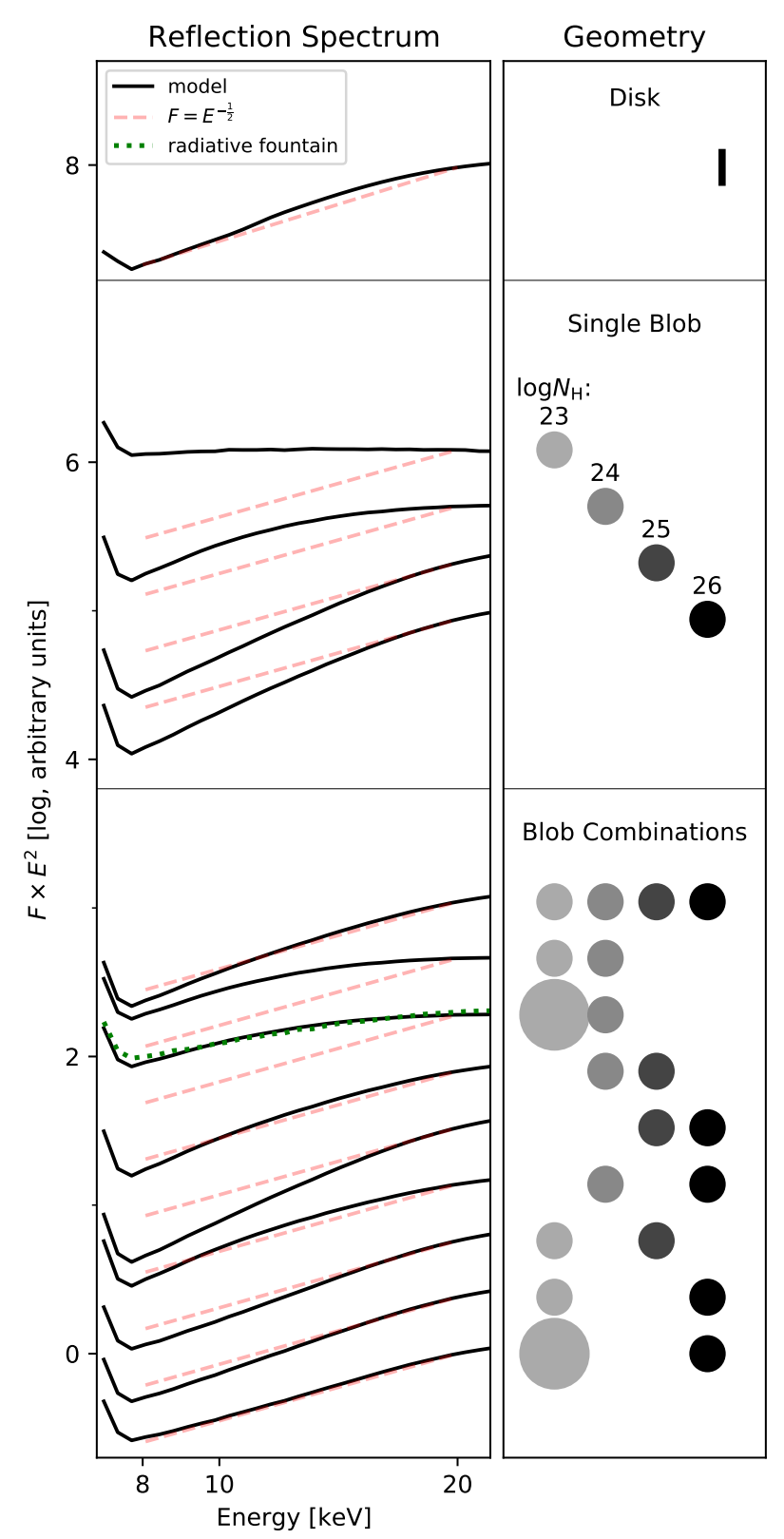

Fig. 8. Reflection spectrum (left, black) for different geometries (right). Black curves are normalised at $20 \mathrm{keV}$, with offsets. The red dashed lines illustrate the observed power law. The top example shows reflection off a semi-infinite disk. The middle four examples show a single blob with column densities $\log N_{\mathrm{H}}=23,24,25$, and 26 . In low-density blobs, the reflection extends to low energies, while for the densest blobs the spectrum rises rapidly (powerlaw photon index $\approx 0$ ). The bottom examples show mixtures of the reflection spectra, assuming equal covering factors in all but the last. Mixtures have a wider Compton hump with a smooth bend. The exceptions are the bottom three examples, which lack the $\log N_{\mathrm{H}}=24$ blob. Their powerlaw-like spectrum approximately follows $F \propto E^{-\frac{1}{2}}$ (red dashed line). The dotted green line shows the radiative fountain model.

(dashed, crossed out arrow in Fig. 9). In contrast, warped disks are one way to provide Compton-thick reflection surfaces that see the corona and the observer unobscured (bottom arrows in Fig. 9). The analysed spectra (Figs. 6 and 8) also place upper limits on reflection from Compton-thin column densities.

Nevertheless, there are substantial caveats to our conclusions. The nuclear reflection on parsec and sub-parsec scales

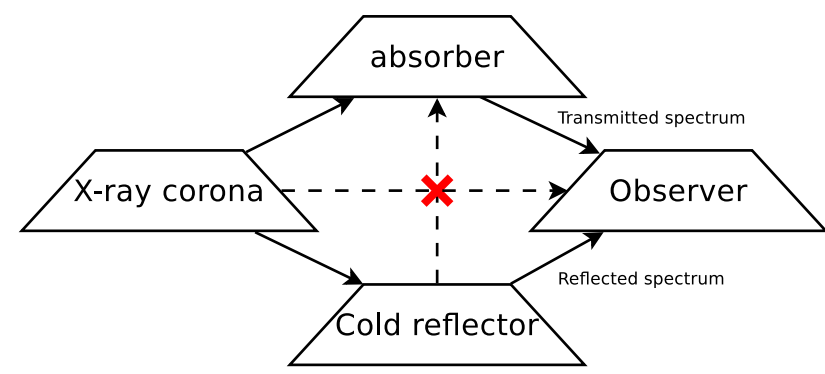

Fig. 9. Inferred unobscured line-of-sights in Compton-thick AGN. Arrows indicate unobscured lines of sight between components. Importantly, the reflection is not reprocessed by the absorber, and the corona is not directly observed (red cross). The reflector is illuminated by the corona directly, not the absorbed emission. The warm mirror is not shown, but would have similar relations as the cold reflector. The host and Milky Way galaxy absorbing screens are also not shown.

needs to be separated by a combination of NUSTAR and Chandra observations. In the future, higher angular resolution missions such as Lynx (The Lynx Team 2018) will provide additional separation. However, also a wider variety of simulations with higher spatial resolution is desired. Additionally, ionisation cone imaging spectroscopy (in optical and X-rays) could be used to constrain the coherence and opening angle of the inner structure (see, e.g., Zhao et al. 2020).

\subsection{Implications and outlook}

The spectral model diversity in the Compton-thick regime has important implications. Firstly, the model choice affects the selection of Compton-thick AGN, and the computation of their space density. Many recent surveys (e.g., Ueda et al. 2014; Buchner et al. 2015; Aird et al. 2017) rely on the spectral model of Brightman et al. (2015). A fixed spectral model is used to compute the sensitivity to detecting Compton-thick AGN as a function of redshift and luminosity. Ricci et al. (2015) explicitly demonstrated that the derived Compton-thick fraction varies with the assumed geometry. Thus, knowing the geometry is crucially important. Secondly, hardness ratios and luminosity absorption corrections in the Compton-thick regime are strongly dependent on the model geometry, in particular when analysing band counts (e.g., $0.5-2 \mathrm{keV}$ and $2-10 \mathrm{keV}$ ) with a fixed spectral model. Recovering the intrinsic accretion luminosity is more reliable when using multi-component spectral fits for each object instead (e.g., Buchner et al. 2014).

Investigation of spectra of nearby AGN suggests evidence of extreme column densities of $N_{\mathrm{H}}>10^{25} \mathrm{~cm}^{-2}$. These are needed either along the LOS (see Table 1) or are present outside the LOS in the models not excluded by the data (see Fig. 6 and Buchner et al. 2019). Such high column densities are outside the range of most previous models. This is in part because the many photon interactions in such dense media are computationally costly to evaluate. XARS provides an efficient implementation for such situations to address this, and can illuminate arbitrarily complex geometries. In part, such high column densities $\left(N_{\mathrm{H}}>10^{25} \mathrm{~cm}^{-2}\right)$ have not been considered because they seem to be rare and unlikely a priori. However, current $\mathrm{X}$-ray surveys of AGN may miss these, as they become strongly absorbed even in the highest energy surveys (e.g., Swift/BAT), and additional multi-wavelength selection methods may need to be developed to complement them. However, strong conclusions require the analysis of a representative and complete 
sample. Systematic NUSTAR follow-up of the Swift/BAT survey is currently the most promising avenue Baloković (2017), with careful multi-component and spatially resolved analyses of NuSTAR, XMM-Newton and Chandra data to separate non-AGN contributions and extended reflection (as in, e.g., Arévalo et al. 2014; Bauer et al. 2015). Because of the complex parameter degeneracies (between geometry parameters, the LOS $N_{\mathrm{H}}$, and source luminosity, $\Gamma$ and $\left.E_{\text {cut }}\right)$, reliable fits require global parameter exploration algorithms such as nested sampling (as, e.g., in Buchner et al. 2014).

Different models retrieve different photon indices in the Compton-thick regime. Even when only $>8 \mathrm{keV}$ data are considered, the effect size, $\Delta \Gamma \approx 0.4$, is substantially larger than the uncertainties (see Table 1). Under the assumption that the accretion proceeds the same at the same Eddington ratio, the photon index distribution should be the same in Compton-thin AGN and Compton-thick AGN. This can provide an additional test of the geometry, even when multiple geometries provide equally good fits per-source. For example, Ueda et al. (2014) find different photon indices for unobscured and moderately obscured AGN, which are not seen in the local Universe when high-energy data are available (Ricci et al. 2017), and may point to an incomplete modelling assumption. A similar test can be done also with the energy cut-off parameter, which can yield low values $\left(E_{\text {cut }}<50 \mathrm{keV}\right)$ in incomplete models.

Acknowledgements. We thank the anonymous referees for constructive suggestions which improved the manuscript. We acknowledge support from the CONICYT-Chile grants Basal-CATA PFB-06/2007 (JB, FEB), ANID grants CATA-Basal AFB-170002 (JB, FEB), FONDECYT Regular 1141218, 1190818 and 1200495 (all FEB), FONDECYT Postdoctorados 3160439 (JB), "EMBIGGEN" Anillo ACT1101 (FEB), and the Ministry of Economy, Development, and Tourism's Millennium Science Initiative through grant IC120009, awarded to The Millennium Institute of Astrophysics, MAS (JB, FEB). This research was supported by the DFG cluster of excellence "Origin and Structure of the Universe". MiB acknowledges support from NASA Headquarters under the NASA Earth and Space Science Fellowship Program, the YCAA Prize Postdoctoral Fellowship, grant NNX14AQ07H, and support from the Black Hole Initiative at Harvard University, which is funded in part by the Gordon and Betty Moore Foundation (grant GBMF8273) and in part by the John Templeton Foundation. KW was supported by JSPS KAKENHI Grant Number 16H03959. This research has made use of the NASA/IPAC Extragalactic Database (NED), which is operated by the Jet Propulsion Laboratory, California Institute of Technology, under contract with the National Aeronautics and Space Administration. This research has made use of SIMBAD (Wenger et al. 2000) and its excellent query API, operated by the Strasbourg astronomical Data Center (CDS).

\section{References}

Aird, J., Coil, A. L., \& Georgakakis, A. 2017, MNRAS, 465, 3390 Anders, E., \& Grevesse, N. 1989, Geochim. Cosmochim. Acta, 53, 197 Antonucci, R. 1993, ARA\&A, 31, 473

Arévalo, P., Bauer, F. E., Puccetti, S., et al. 2014, ApJ, 791, 81

Baloković, M. 2017, PhD Thesis, California Institute of Technology, USA Baloković, M., Comastri, A., Harrison, F. A., et al. 2014, ApJ, 794, 111

Baloković, M., Brightman, M., Harrison, F. A., et al. 2018, ApJ, 854, 42 Bauer, F. E., Arévalo, P., Walton, D. J., et al. 2015, ApJ, 812, 116 Bianchi, S., Guainazzi, M., \& Chiaberge, M. 2006, A\&A, 448, 499 Brightman, M., \& Nandra, K. 2011, MNRAS, 413, 1206 Brightman, M., Nandra, K., Salvato, M., et al. 2014, MNRAS, 443, 1999 Brightman, M., Baloković, M., Stern, D., et al. 2015, ApJ, 805, 41 Buchner, J., \& Bauer, F. E. 2017, MNRAS, 465, 4348
Buchner, J., Georgakakis, A., Nandra, K., et al. 2014, A\&A, 564, A125 Buchner, J., Georgakakis, A., Nandra, K., et al. 2015, ApJ, 802, 89 Buchner, J., Schulze, S., \& Bauer, F. E. 2017, MNRAS, 464, 4545 Buchner, J., Brightman, M., Nandra, K., Nikutta, R., \& Bauer, F. E. 2019, A\&A, 629, A16

Chan, C.-H., \& Krolik, J. H. 2016, ApJ, 825, 67

Dorodnitsyn, A., \& Kallman, T. 2017, ApJ, 842, 43

Fabbiano, G., Paggi, A., Karovska, M., et al. 2018, ApJ, 855, 131

Fujimoto, A., Tanaka, T., \& Iwata, K. 1986, IEEE Comput. Graph. Appl., 6, 16 Furui, S., Fukazawa, Y., Odaka, H., et al. 2016, ApJ, 818, 164

Gallagher, S. C., Everett, J. E., Abado, M. M., \& Keating, S. K. 2015, MNRAS, 451, 2991

Gandhi, P., Lansbury, G. B., Alexander, D. M., et al. 2014, ApJ, 792, 117

Gaspari, M., Brighenti, F., \& Temi, P. 2015, A\&A, 579, A62

Greenhill, L. J., Booth, R. S., Ellingsen, S. P., et al. 2003, ApJ, 590, 162

Greenhill, L. J., Tilak, A., \& Madejski, G. 2008, ApJ, 686, L13

Hopkins, P. F., Hayward, C. C., Narayanan, D., \& Hernquist, L. 2012, MNRAS, 420,320

Ikeda, S., Awaki, H., \& Terashima, Y. 2009, ApJ, 692, 608

Izumi, T., Wada, K., Fukushige, R., Hamamura, S., \& Kohno, K. 2018, ApJ, 867, 48

Jud, H., Schartmann, M., Mould, J., Burtscher, L., \& Tristram, K. R. W. 2017, MNRAS, 465, 248

Lawrence, A., \& Elvis, M. 2010, ApJ, 714, 561

Liu, Y., \& Li, X. 2014, ApJ, 787, 52

Magdziarz, P., \& Zdziarski, A. A. 1995, MNRAS, 273, 837

Maloney, P. R., Begelman, M. C., \& Pringle, J. E. 1996, ApJ, 472, 582

Marinucci, A., Miniutti, G., Bianchi, S., Matt, G., \& Risaliti, G. 2013, MNRAS, 436, 2500

Markowitz, A. G., Krumpe, M., \& Nikutta, R. 2014, MNRAS, 439, 1403

Masini, A., Comastri, A., Baloković, M., et al. 2016, A\&A, 589, A59

Matt, G., Pompilio, F., \& La Franca, F. 1999, New Astron., 4, 191

Matt, G., Fabian, A. C., Guainazzi, M., et al. 2000, MNRAS, 318, 173

Mościbrodzka, M., \& Proga, D. 2013, ApJ, 767, 156

Murphy, K. D., \& Yaqoob, T. 2009, MNRAS, 397, 1549

Nandra, K., \& Pounds, K. A. 1994, MNRAS, 268, 405

Nandra, K., O'Neill, P. M., George, I. M., \& Reeves, J. N. 2007, MNRAS, 382 , 194

Nenkova, M., Sirocky, M. M., Ivezić, Ž., \& Elitzur, M. 2008, ApJ, 685, 147

Netzer, H. 2015, ARA\&A, 53, 365

Oh, K., Koss, M., Markwardt, C. B., et al. 2018, ApJS, 235, 4

Petterson, J. A. 1977a, ApJ, 214, 550

Petterson, J. A. 1977b, ApJ, 216, 827

Pringle, J. E. 1996, MNRAS, 281, 357

Ramos Almeida, C., \& Ricci, C. 2017, Nat. Astron., 1, 679

Ricci, C., Ueda, Y., Koss, M. J., et al. 2015, ApJ, 815, L13

Ricci, C., Trakhtenbrot, B., Koss, M. J., et al. 2017, ApJS, 233, 17

Risaliti, G., Elvis, M., \& Nicastro, F. 2002, ApJ, 571, 234

Rivers, E., Markowitz, A., \& Rothschild, R. 2013, ApJ, 772, 114

Schartmann, M., Mould, J., Wada, K., et al. 2018, MNRAS, 473, 953

Tanimoto, A., Ueda, Y., Odaka, H., et al. 2019, ApJ, 877, 95

Teng, S. H., Rigby, J. R., Stern, D., et al. 2015, ApJ, 814, 56

The Lynx Team 2018, ArXiv e-prints [arXiv:1809. 09642]

Tristram, K. R. W., Burtscher, L., Jaffe, W., et al. 2014, A\&A, 563, A82

Turner, T. J., George, I. M., Nandra, K., \& Mushotzky, R. F. 1997, ApJ, 488, 164

Ueda, Y., Akiyama, M., Ohta, K., \& Miyaji, T. 2003, ApJ, 598, 886

Ueda, Y., Akiyama, M., Hasinger, G., Miyaji, T., \& Watson, M. G. 2014, ApJ, 786, 104

Verner, D. A., Ferland, G. J., Korista, K. T., \& Yakovlev, D. G. 1996, ApJ, 465, 487

Wada, K. 2012, ApJ, 758, 66

Wada, K. 2015, ApJ, 812, 82

Wada, K., Schartmann, M., \& Meijerink, R. 2016, ApJ, 828, L19

Wada, K., Yonekura, K., \& Nagao, T. 2018a, ApJ, 867, 49

Wada, K., Fukushige, R., Izumi, T., \& Tomisaka, K. 2018b, ApJ, 852, 88

Wenger, M., Ochsenbein, F., Egret, D., et al. 2000, A\&AS, 143, 9

Williamson, D., Hönig, S., \& Venanzi, M. 2019, ApJ, 876, 137

Zhao, X., Marchesi, S., Ajello, M., Baloković, M., \& Fischer, T. 2020, ApJ, 894, 71 


\section{Appendix A: 3D Discrete digital analyser}

We briefly describe how density grids were illuminated and processed into X-ray spectra. In XARS (see Buchner et al. 2019), the user needs to specify two functions, one describing the geometry, and the second specifying how escaping photons are gridded (e.g., in viewing angle). For the latter we chose gridding in viewing angle and LOS column density to the corona (see Buchner et al. 2019), as these are the primary aspects affecting the output spectrum. The custom geometry function receives a photon position, direction and $N_{\mathrm{H}}$ distance (in optical depth units) to travel until the photons stops (for interaction with the medium).

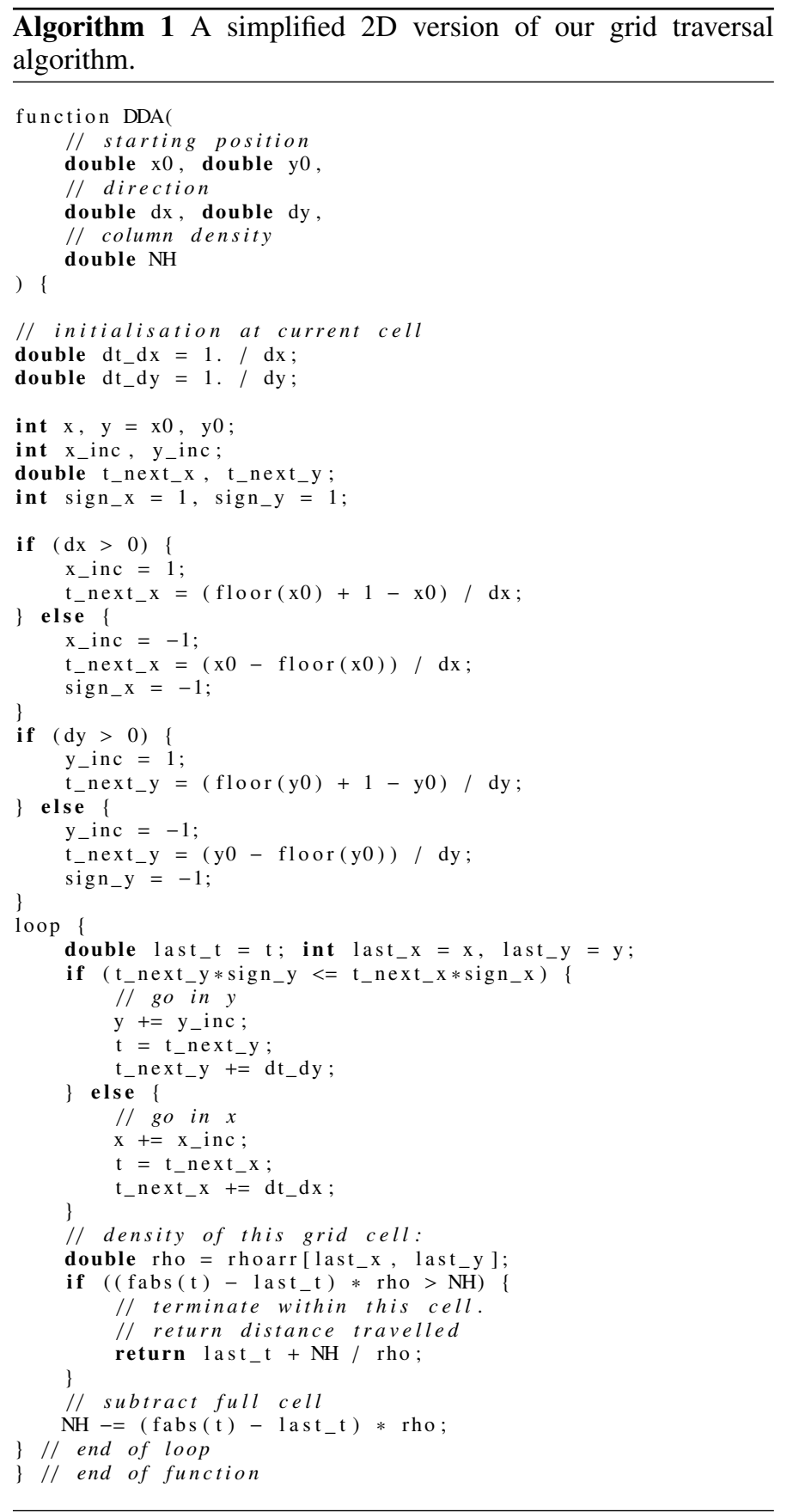

In the case of our density grids, the density within a grid cell is assumed to be constant. The problem then transforms to measuring the distances traversed in each grid cell and multiplying

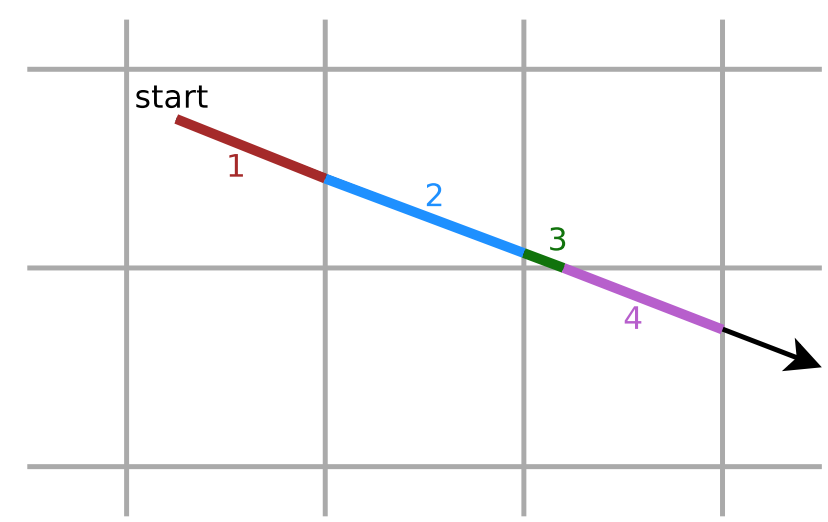

Fig. A.1. Illustration of photon propagation in a grid. The Discrete Digital Analyser algorithm finds the sequence of points where the line crosses grid boundaries. This allows segmentation of the line by grid cell, illustrated by the numbers and colours.

with the grid density to obtain the grid cells $N_{\mathrm{H}}$. We illustrate the segmentation of the path in Fig. A.1. This is subtracted from the target $N_{\mathrm{H}}$ distance until it reaches zero. Measuring the distances traversed in each grid cell is solved with a 3D Discrete Digital Analyser, an algorithm developed for drawing lines through pixels in computer graphics (Fujimoto et al. 1986). We present a two-dimensional simplified version in Fig. 1; the extension to three dimensions is straight-forward. The algorithm computes the step size between grid lines given the current direction, and then steps through the grid cells in the right order (either in $x$ or $y$ ). The outlined algorithm returns the coordinate space distance traversed until the photon has travelled through a column density of $N_{\mathrm{H}}$.

\section{Appendix B: Spectral fits of NGC 3393 and NGC 424}

For completeness, we also present spectral fits for the two other galaxies, NGC 3393 and NGC 424. The setup is virtually identical to that described in Sect. 4.4. The only difference in the modelling is that no supernova remnant contaminant is included.

Figure B.1 presents the spectral fit of five models. All models give good fit qualities $\left(\chi^{2} /\right.$ d.o.f. $\left.<80 / 77\right)$. However, the best fit

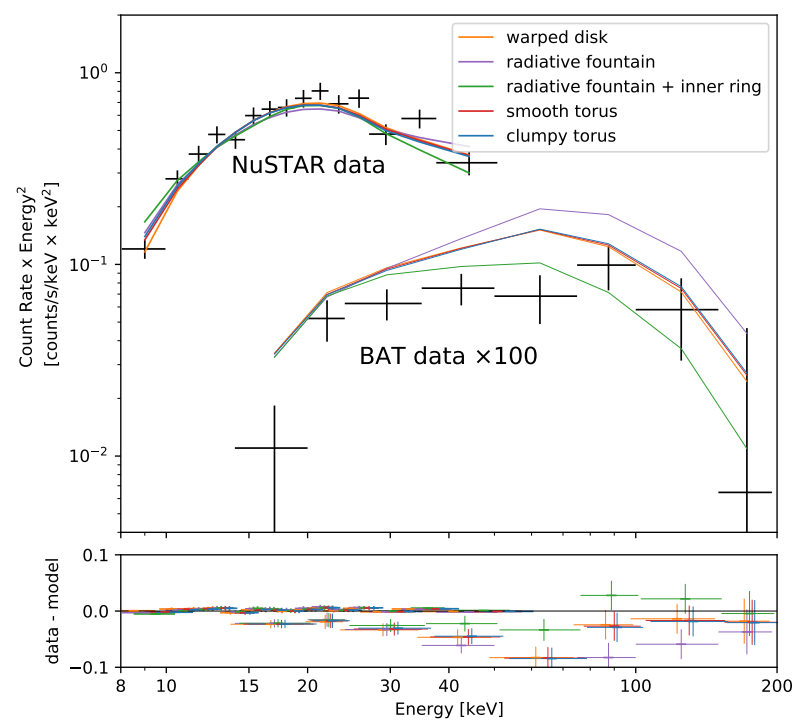

Fig. B.1. Same as Fig. 7, but for NGC 3393. 


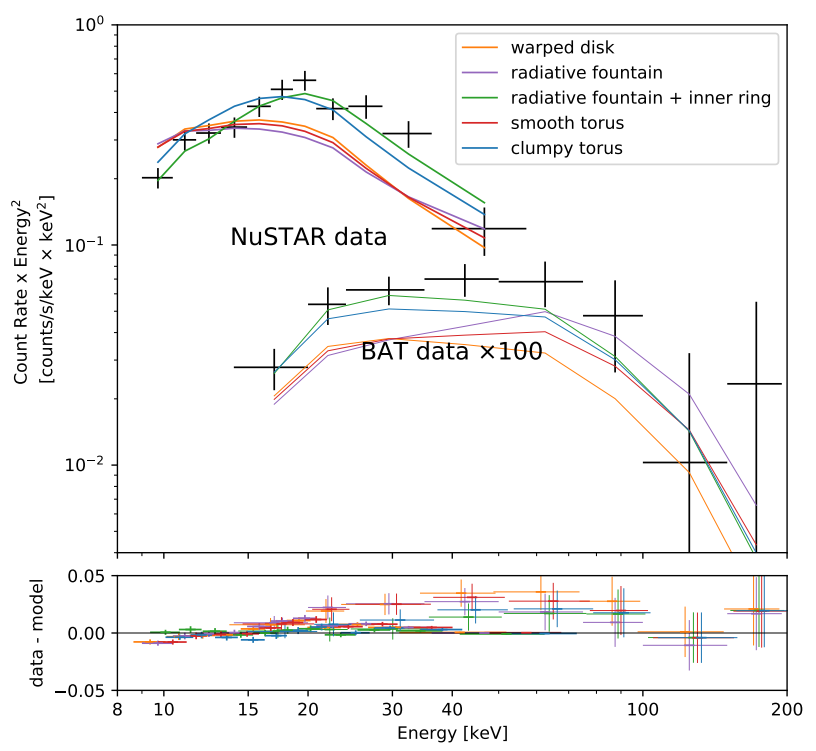

Fig. B.2. Same as Fig. 7, but for NGC 424. photon indices of the clumpy model and the radiative fountain model are low $(1.69 \pm 0.11$ and $1.53 \pm 0.16$, respectively). For the other models, including the radiative fountain model with inner ring, the best fit photon index has values more typical of the population (the 1.8-2.0 interval overlaps the $1 \sigma$ confidence intervals). All model fits produce best-fit column densities in the Compton-thick regime.

Figure B.2 presents the equivalent spectral fit for NGC 424. All models give good fit qualities $\left(\chi^{2} /\right.$ d.o.f. $\left.\lesssim 115 / 128\right)$. However, the best fit photon indices of the clumpy torus model and the smooth torus model are high $(2.46 \pm 0.27$ and $2.45 \pm 0.26$, respectively). For the other models, the best fit photon index have values typical of the population (2.0 is within the $1 \sigma$ confidence intervals). All model fits produce best-fit column densities in the Compton-thick regime.

The spectral fits confirm the point of Fig. 6: The spectra can be explained by an AGN with a typical photon index $\Gamma=2$ under the warped disk model, but not under the radiative fountain model, unless a inner ring is added. 